\title{
Impervious surface extraction of multi-source high-resolution images in 2018 in Anhui Province
}

\author{
Wang Fan ${ }^{1}$, Jia Xiaofeng, ${ }^{2, *}$ Li Mengya ${ }^{2}$ and Jia Shijie ${ }^{3}$ \\ ${ }^{1}$ Anhui Xinhua University, Hefei ,230088, China \\ ${ }^{2}$ School of Surveying and Mapping Science and Engineering, Shandong University of Science and Technology, Qingdao 266510, China \\ ${ }^{3}$ School of Civil Engineering and Architecture, Shandong University of Technology, Zibo, Shandong 255000, China
}

\begin{abstract}
With the development of the economy and the continuous improvement of social demand, the impervious area is more and more representative of the urbanization process and economic development level of the society. Anhui Province is a big province, and the impervious information is an essential element for an accurate understanding of economic development.To accurately understand the impervious surface and economic development level of Anhui Province, this study selects the training samples of Anhui province, trains the classifier, uses the classification algorithm of support vector machine, combines 2018 Sentinel-2 and Landsat8 in Matlab, and uses the LUOJIA-1 nighttime light data as auxiliary. The data was used to make land use planning maps for forests, farmland, impervious surfaces and water bodies in Anhui Province, and then the impervious information of the province in Anhui Province was extracted with high precision in 2018. Compared with the results of Sentinel-2\&LUOJIA-1 and Landsat8\& LUOJIA-1, it is proved that the combination of Sentinel-2, Landsat8 and LUOJIA-1 data can extract impervious information with high accuracy and high precision.
\end{abstract}

\section{Introduction}

With the development of urbanization and social and economic development, monitoring of urban expansion based on remote sensing images is particularly important. Therefore, effective monitoring of urban expansion on a regional scale can provide scientific reference for land planning and ecological environmental protection, and has important practical significance [1]. At present, many scholars in China have studied urban expansion. Chang Bianrong and Li Rendong analyzed the spacetime characteristics of construction land expansion in Wuhan [2], using object-oriented classification method to extract construction land [2], using ESI, EII, EDI and SDE spatial analysis method shows that the early expansion has evolved to the northeast-southwest direction [2], and there is no directional conclusion in the later stage [2], which provides a basis for the decisionmaking of various government agencies in the later period; Chen Zheng et al. For example, the remote sensing image data and nighttime lighting data were used to extract town information [1], distinguish between urban land and non-urban land, and analyze the characteristics of urban space-time expansion [1]; Sun Shanlei and others take the Hangzhou Bay area as an example. The combination of decision tree and shape index to extract urban land, and they use information proves the feasibility of this method, and Hangzhou City expands to the northwest and north directions [3]. This research is intended to explore the accuracy, so the use of support vector machine classification algorithm, fusion of different data source bands, improve classification accuracy. The detailed process is shown in Figure 1.

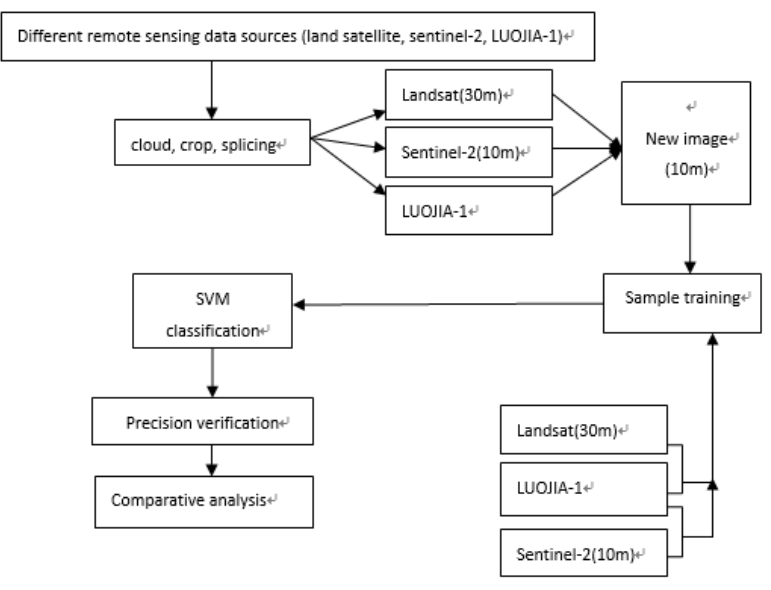

Fig. 1. flow chart

\section{Data and methods}

\subsection{Introduction to the study area}

Anhui Province is located in the eastern part of China, bordering Shandong, Jiangsu, Henan, Hubei and other provinces. There are 16 prefecture-level cities including Hefei ,Wuhu, Maanshan, Tongling, Chizhou, Xuancheng

\footnotetext{
Corresponding author: jxf2352652371@163.com
} 
and Huangshan. Anhui Province is the central economic zone, the hinterland of East China, connecting North Union South and connecting to the east and west. Anhui Province is located in the eastern monsoon region of China. Because the Huaihe River passes through it, the north of the Huaihe River belongs to the temperate monsoon climate, and the south of the Huaihe River belongs to the subtropical monsoon climate. The terrain of Anhui is diverse, with plains (44.7\%), mountains (27.8\%), and hills (27.5\%). The total land area of Anhui Province is 139,600 square kilometers, and the types of land resources are diverse. The paddy fields, dry land, forest land and grassland all have large areas, and the land composition is reasonable and the land use potential is very large. Figure 2 shows the scope of the study area. The base map is the 2018 Google Image.

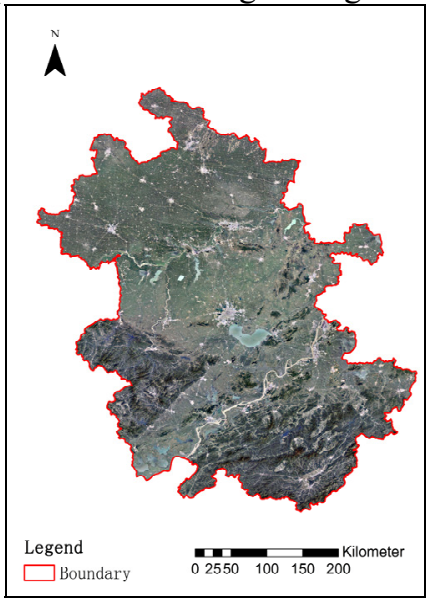

Fig. 2. Study area

\subsection{Data and preprocessing}

The study used 2018 nighttime lighting data (LUOJIA1) ,Sentinel-2 and Landsat8 in Anhui Province. Sentinel2 data is downloaded from the official website of ESA (https://scihub.copernicus.eu/dhus/\#/home).The coverage of this study is 2018, and the 2nd, 3rd, 4th and 8th bands are selected. The resolution is 10 meters. Landsat 8 was obtained from the USGS official website (https://earthexplorer.usgs.gov/). The time is consistent with the Sentinel-2 data. The green, red, near-infrared and mid-infrared bands are selected, and the spatial resolution is $30 \mathrm{~m}$. "LUOJIA-1" is the world's first professional night-time remote sensing satellite. It is jointly developed and produced by the Wuhan University team and related institutions. At 12:13 on June 2, 2018, the the NO.6 of high resolution and its carrying the scientific test satellite, carried by the Long March II carrier rocket, was launched at the Jiuquan Satellite Launch Center in China, and the satellite entered the orbit. The LUOJIA-1 is equipped with a high-sensitivity luminous camera with an accuracy of 100 meters on the ground. Multi-source data completes pre-processing by performing cloud removal, calibration, geometric correction, splicing, and cropping on different data sources.

\subsection{Support vector integral class method}

\subsubsection{Training plot selection}

Training sample selection should be representative and should be evenly selected. This study selected four types of land types: forest, farmland, water body and impervious surface. Detailed statistics are shown in Table 1.

Table 1. Training plot statistics

\begin{tabular}{|c|c|}
\hline Feature type & $\begin{array}{c}\text { Number of training } \\
\text { plots/piece }\end{array}$ \\
\hline Impervious surface & 132 \\
\hline water & 140 \\
\hline forest & 176 \\
\hline cropland & 96 \\
\hline
\end{tabular}

\subsubsection{Band calculation and classification}

Table 2. Index calculation table

\begin{tabular}{|c|c|l|}
\hline $\begin{array}{c}\text { Source } \\
\text { of data }\end{array}$ & Selected band & \multicolumn{1}{|c|}{$\begin{array}{c}\text { Characteristic index } \\
\text { calculation }\end{array}$} \\
\hline & & $\begin{array}{l}\text { NDVI=(NIR- } \\
\text { RED)/(NIR+RED) } \\
\text { NDWI=(GREEN- } \\
\text { NIR)/( GREEN+NIR) }\end{array}$ \\
& & NDBI=(MIR- \\
& NIR)/(MIR+NIR) \\
& NIR,MIR & NDVI=(NIR- \\
& & RED)/(NIR+RED) \\
\hline & & NDWI=(GREEN- \\
& & NIR)/( GREEN+NIR) \\
Sentinel-2 & GREEN,RED, & NDBI (SWIR- \\
& NIR,SWIR & NIR)/(SWIR+NIR) \\
& & \\
\hline
\end{tabular}

According to the statistics in Table 2, the green light band, red light band, near-infrared and mid-infrared bands of Landsat8 were selected for exponential calculation. The calculated feature index has Normalized Vegetation Index (NDVI) and Normalized Water-body Index ( NDWI), Normalized Building Index (NDBI), and selected red and near infrared bands. At the same time, the Sentinel-2 data space resolution of 10 meters is selected, which is the 3rd (GREEN), 4 (RED), and 8 (NIR) bands respectively. The SWIR band of the sentinel data is better for building extraction. Ok, so the 11 (SWIR) band with a resolution of 20 meters was selected and NDVI, NDBI, NDWI were calculated.

Support Vector Machine Classification (SVM) is a model for supervised classification. Data can be analyzed, patterns identified, and used for classification and regression analysis. The support vector machine method is based on the VC dimension theory and structural risk minimization principle of statistical learning theory. It seeks the best compromise between the complexity of the model (ie, the learning accuracy of a particular training sample) and the ability to learn (ie, the ability to identify any sample without error) based on limited sample information, in order to obtain the best promotion ability. The SVM used in the research can not 
only classify the cell attributes, but also solve the problem of interval values.

RED, NIR, NDVI, NDWI, NDBI of Landsat8 and LUOJIA-1 data were added to the SVM algorithm for classification. And RED, NIR, NDVI, NDWI, NDBI of Sentinel-2 and LUOJIA-1 data were added to the SVM algorithm for classification. Finally, all three kinds of data are applied to the classification. What's more, Comparing and analyzing the three results

\section{Results and analysis}

Landsat8's RED, NIR, NDVI, NDWI, NDBI and Sentinel-2 data were combined with RED, NIR, NDVI, NDWI, and NDBI to generate a 10-meter map of land use in Anhui Province. Landsat8's RED, NIR, NDVI, NDWI, NDBI and RED1 data and Sentinel-2 data for RED, NIR, NDVI, NDWI, NDBI and LUOJIA-1 data each generate 10 meters of Anhui Province land use classification map results As shown in Figure 3.

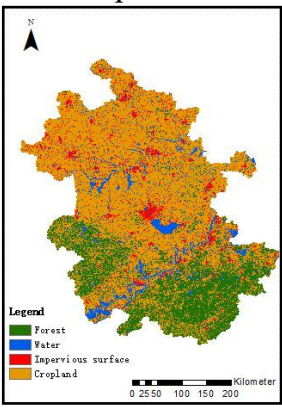

(A)Landsat\&Sentine 1-2\&LUOJIA-1

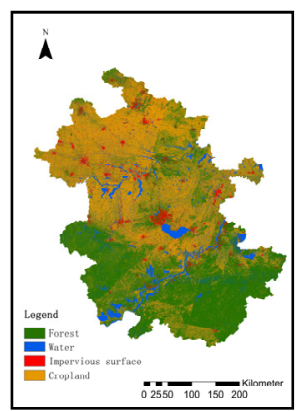

(C)Landsat\& LUOJIA-1

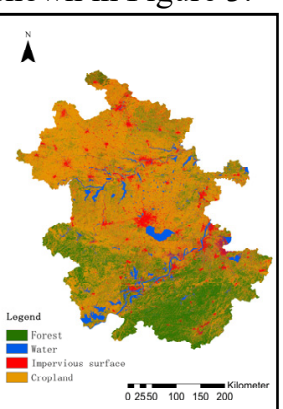

(B)Sentinel2\&LUOJIA-1 ig. 3. Multi-source data classification result graph

Based on the SVM classification results, the impervious surface extraction results are shown in Figure 4.

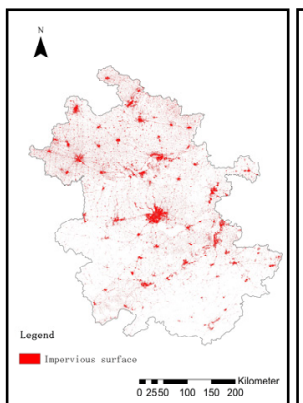

(A)Landsat\&Sentine 1-2\&LUOJIA-1

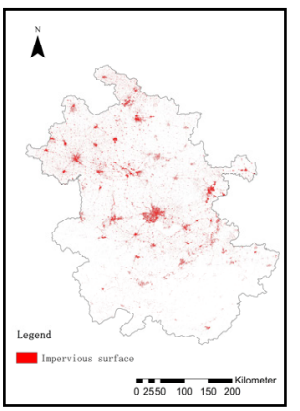

(C)Landsat\& LUOJIA-1

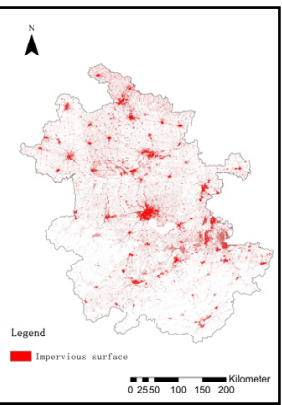

(B)Sentinel2\&LUOJIA-1
Fig. 4. .Multi-source data impervious surface extraction results

By selecting sample points in Google Earth and using the confusion matrix to verify the classification results, Figure 5 shows the accuracy verification points selected by Google. The verification results are shown in Table 3 below.

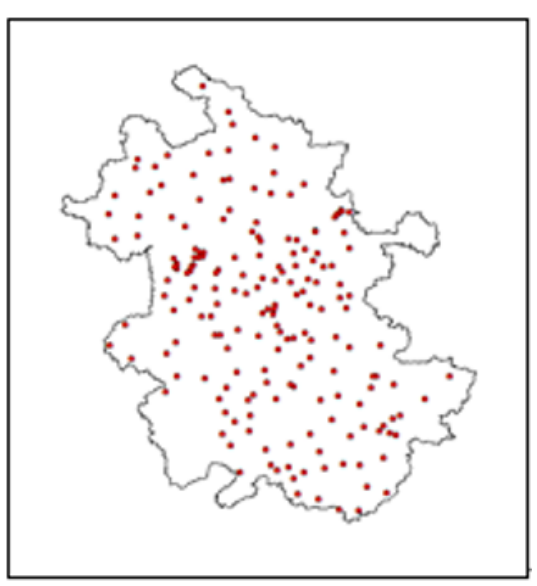

Fig. 5. accuracy verification points

Table 3. Multi-source data source accuracy verification results and impervious surface area statistics

\begin{tabular}{|c|c|c|}
\hline Source of data & Accurate & $\mathrm{Area}\left(\mathrm{km}^{2}\right)$ \\
\hline $\begin{array}{c}\text { Sentinel- } \\
\text { 2\&Landsat8\&LUOJIA-1 }\end{array}$ & 0.97 & 19194.283434 \\
\hline Landsat 8\&LUOJIA-1 & 0.93 & 3310.740774 \\
\hline Sentinel-2\&LUOJIA-1 & 0.94 & 9497.815011 \\
\hline
\end{tabular}




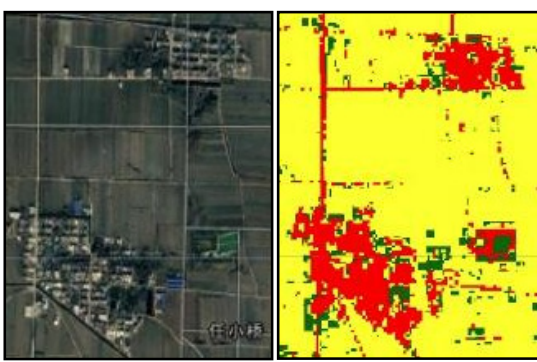

(A)Google image (B)Landsat\&Sentinel -2\&LUOJIA-1

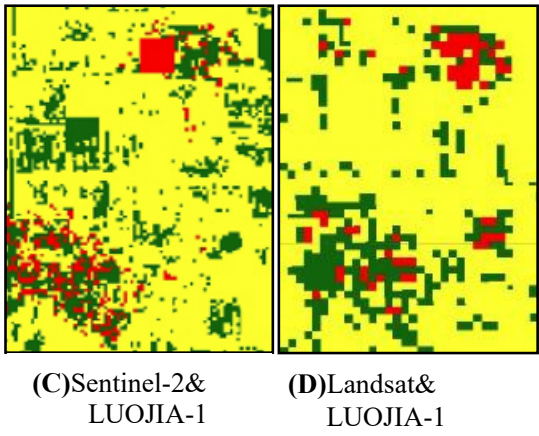

Fig. 6. Three data source small village identification map

Through the analysis of statistics in Figure 3, Figure 4 and Table 3, for the extraction of the impervious surface of the entire province of Anhui Province in 2018, the data of different resolutions of Landsat, Sentinel-2 and LUOJIA-1 are used, with an accuracy of 0.97 . The accuracy is high, and the area of the impervious surface is $19194.283434 \mathrm{~km}^{2}$, and the area is also accurate. The classification accuracy of Landsat 8\&LUOJIA-1 and Sentinel-2\&LUOJIA-1 is lower than that of Landsat\&Sentinel-2\&LUOJIA-1, and the area statistics are quite different.

As can be seen from Figure 6, using three different data source classifications, based on the improvement of accuracy, as shown in Figure 6 (B), the combination of Landsat \& Sentinel-2 \&LUOJIA-1 can be located in the field or mountain village. The village and the road are accurately extracted. As in (C), Sentinel-2 \&LUOJA-1 is not able to extract this type of feature. In Figure (D), Landsat 8 \&LUOJIA-1 will be misclassified, and it is easy to divide the village into forest land.

\section{Conclusion}

Based on the 2018 multi-source high-resolution remote sensing imagery to extract the impervious surface of Anhui Province, it can be seen from the extraction accuracy that the Sentinel-2 data, the Landsat data and the LUOJIA-1 data combination have 11 bands to the impervious surface. The extraction accuracy is the highest. Similarly, the impervious surface area of Anhui Province is closer to $19194.283434 \mathrm{~km}^{2}$, and the three data sources combine to extract the impervious surface. The extraction effect on small villages is better. In contrast, when only Landsat8\&LUOJIA-1 data or only Sentinel-2\&LUOJIA-1 data is used, small villages are difficultly identified as forest land or farmland, and the error is greatly affected. In addition, the map of the impervious surface of Anhui Province generated in the study has higher precision and higher resolution. Therefore, the research method is practical. With the advancement of National land survey, the accuracy and efficiency of remote sensing image interpretation are increasingly important. Multi-source high-resolution remote sensing image classification and extraction based on SVM method can meet the characteristics of today's social accuracy and efficiency, and can coordinate and save manpower, material resources and financial resources.

\section{References}

1. Chen Meiqiu, Zhao Xiaomin, Wu Cifang. Advances in Remote Sensing Monitoring of Urban Land Expansion[J]. Journal of Jiangxi Agricultural University, 1999(02): 97-101

2. Chang Biangrong, Li Rendong Remote Sensing Monitoring and Spatial Differentiation Analysis of Construction Land Expansion in Wuhan [J] Remote Sensing of Land and Resources, 2017, 29(03): 118123.

3. Sun Shanlei, Zhou Suozhen, Wei Guoshuan, Ji Zongwei, Chen Hongmei. Remote Sensing Dynamic Monitoring of Urban Expansion in Hangzhou Bay Area,J.N.R 2,327-335(2008)

4. Chen Benqing. Dynamic monitoring and analysis of urbanization process and environmental changes in Xiamen City using multi-temporal satellite remote sensing images [d]. Fuzhou University, 2002.

5. LI Xing-hua,LI Yun-peng,YANG Li-ping.Study on Comprehensive Application of Drought Monitoring and Evaluation Methods in Inner Mongolia[J].Journal of Arid Land Resources and Environment,2014,28(03):162-166.

6. Yue Shengru. Analysis and application of remote sensing monitoring method for soil moisture content in Inner Mongolia pastoral area based on NDVI partition [D]. Inner Mongolia Agricultural University, 2015.

7. J. Lei. Remote sensing monitoring of desertification degree and its vegetation characteristics and soil nutrient analysis in Zoige grassland,D,Sichuan Agricultural University(2012)

8. Zhang Tingting. Remote Sensing Monitoring and Salinization Process Spatial Analysis and Evaluation of Land Salinization Pattern in the Yellow River Delta [D]. Fudan University, 2011.

9. Zhang Wenjun. Remote Sensing Dynamic Characteristics Monitoring and Prediction Analysis of Landslide Hazards[D]. Southwest Jiaotong University, 2007.

10. Zhang Hua. Environmental Monitoring and Analysis Based on Remote Sensing and GIS [D]. Hunan University, 2004. 